\title{
APRENDIZAJE BASADO EN PROYECTOS Y SU EVALUACIÓN FORMATIVA A TRAVÉS DE RÚBRICAS
}

Project-based learning and its assessment through rubrics

Aprendizagem baseada em projetos e sua avaliação através da rubricas

\author{
Juan Fraile \\ Universidad Francisco de Vitoria, España. \\ e-mail: juan.fraile@ufv.es
}

\begin{abstract}
Resumen
Esta experiencia describe un em pleo formativo de la rúbrica com o instrumento de evaluación y calificación para un proyecto grupal implementado a lo largo de una asignatura del grado en Ciencias de la Ac tividad Física y del Deporte. A medida que se fueron trabajando los contenidos del curso, se fue implicando a los estudiantes en la elaboración de criterios con el fin de cocr ear la rúbrica. En consecuencia, a lo largo del curso se fueron incorporando más criterios asociados a lo $\mathrm{s}$ diferentes bloques. Se implementó el empleo de la App Trello pa ra la organización interna de cada grupo. Asimismo, durante las clases, se fue realizando el trabajo y actividades metacognitivas para avanzar en su comprensión y aprendizaje.

Palabras clave: Rúbrica; autorregulación; aprendizaje basado en proyectos
\end{abstract}

\begin{abstract}
This experience describes a formative use of the rubric as an assessment and grading tool for a group project im plemented throughout a subject of the degree in Sports Sciences. As the contents of the course were managed, the students were involved in the elaboration of criteria to co-create the rubr ic. As a result, more standards associated with the different blocks were incorporated throughout the course. The Trello App was implemented for the internal organisation of each group. Also, during the lessons, work on metacognitive activities was carried out to improve their understanding and learning. Keywords: Rubric; self-regulation; project-based learning
\end{abstract}




\section{Resumo}

Este descreve uma experiência formativa usando a rubrica com o uma ferramenta para avaliação e qualificação para um projeto de grupo implementado ao longo de um curso de licenciatura em Ciências da Atividad e Física e do Desporto. Como eles estavam trabalhando o conteúdo do curso, foi e nvolver os alunos no desenvolvim ento de critérios, a fi m de co-cri ar a seção. Por conseguinte, durante o curso que foram incorporados vários critérios asso ciados com os diferentes blocos. o uso do App Trello para a organização interna de cada grupo foi implementado. Também durante as aulas, ele estava fazendo o trabalho e as ativ idades metacognitivas para avançar s ua compreensão e aprendizagem.

Palavras-chave: Rubrica; Auto-regulação; Aprendizagem baseada em projetos

\section{Introducción}

El empleo formativo de las rúbricas puede mejorar el aprendizaje y el rendim iento académico (Panadero \& Jonsson, 2013). A través d e éstas, se puede prom over la autoevaluación con el fin de que los estudian tes identifiquen sus aciertos y errores y, en consecuencia, mejoren sus tareas (K ostons, van Gog \& Paas , 2012). Un paso m ás allá en el em pleo de es te instrumento, además de la im plementación de activ idades metacognitivas, es la cocreación de la rúbrica entre estudiantes y docente con el objetivo de maximizar los beneficios de su empleo, especialmente en cuanto a la autorregulación (Fraile, Panadero, \& P ardo, 2017). Por tanto, el objetivo de esta experiencia es la implementación de los procesos de autorregulación a través del empleo de rúbricas y el uso de la app Trello.

\section{Contextualización}

La experiencia se lleva a cabo en la asignatura "Actividades deportivas en la naturaleza: ocio y recreación deportiva" del tercer curso d el grado en Ciencias de la Actividad Física y del Deporte. E sta es una asignatu ra de 6 créditos con unos 80 alum nos que acuden presencialmente divididos en 4 grupos a cargo de dos docentes. Uno de los objetivos fundamentales de la asignatura, y el principal descrito en esta experiencia, es el diseño de una carrera de orientación o ex cursión y todos los aspectos organizativos y relacionados con el medio natural asociados a ésta. 


\section{Diseño y desarrollo}

Esta experiencia se encuadra en un trabajo grupal (máximo 4 estudiantes) asociado con el $50 \%$ de la calificación final de la asignatura. Este trabajo se realiza a lo largo de toda la asignatura y se entrega al final d e ésta siendo el objetivo que los co ntenidos que se van viendo a lo largo del curso se vayan incorporando al trabajo.

A su vez, existen 4 opciones que los es tudiantes pueden elegir relacionado con llevar a cab o una evalu ación auténtica y la experiencia diseñada a la realidad. $\mathrm{L}$ as opciones son las siguientes:

- Para optar al $100 \%$ de la calificación de 1 trabajo los estudiantes deben diseñar una carrera de orientación y llevarla a cabo con al menos 15 participantes que no pueden ser sus compañeros de clase.

- Con el fin de optar al $80 \%$, deben dise ñar una excursión en el m edio natural guiando a 10 participantes.

- Para optar al $60 \%$, únicam ente tendrán que hacer el diseño de una carrera de orientación, sin llevarla a cabo.

- Para el 50 \%, diseñar una excursión en el medio natural, sin llevarla a cabo.

La secuencia, en cuanto a trabajar los conten idos vistos en clase y relacionarlos con el trabajo, es la siguiente:

1. Trabajo de los contenidos a través de lección magistral, aprendizaje basado en la investigación y otras metodologías activas.

2. Elaboración de criterios sobre los c ontenidos vistos $\mathrm{y}$-en algunos casosimplicación en la elab oración de las de finiciones de calidad de la rúbrica. Después, con las aportaciones de todos lo s grupos, se modificará la rúbrica que los profesores proporcionan a los estudiantes.

3. Se proporciona la rúbrica elaborada previam ente, sólo de esa parte, para que los estudiantes la trabajen y acoplen a su trabajo. Se diseña una clase en estaciones para poder atenderles de forma más individual, explicándoles la rúbrica y qué se espera.

La rúbrica definitiva - una para cada tipo de trabajo: orientación o excursión- se compone de 24 y 23 criterios. Cada uno de los criterios tiene un peso diferente, 
establecido por los docentes, en base a la im portancia otorgada. La suma de todos los puntos posibles serían 28 y 26,5 en función del tipo de trabajo. Además, los docentes a la hora de calificar el trabajo tenían la posib ilidad de sumar o restar puntos en el rango de más/menos 6 a la puntuación obtenida.

Además, se trabajó el reparto de tareas concretas dentro de cada grupo a través de la App Trello y las lis tas en cuanto a tareas "pen dientes", "en proceso" o "terminadas". Los docentes prepararon un tabl ero para cada equipo y ciertas tarjetas iniciales que compartieron con cada grupo a través de un link que enviaron al grupo de Whatsapp que se tiene con los estudiantes. De esta form a, a través de sus m óviles y ordenadores, se podían dar de alta en la App y tener asoc iado directamente el tablero creado para ellos.

Tras las vacaciones de Se mana Santa, se solicitará (todavía no se ha llevado a cabo) la entrega parcial del trabajo sin im portar su estado. Los docentes lo evaluarán con la rúbrica, registrando un feedback de algunos criterios. En una de las sesiones se pedirá a los alum nos que se autoevalúe $n$ en grupo y después se les dará la retroalimentación realizada por los docentes. En una de las es taciones este d ía, se dialogará con los grupos uno por uno.

El trabajo lo podrán seguir enviándolo a los docentes hasta la última sesión de la asignatura para poder recibir retroalim entación. La entrega final será dos sem anas después coincidiendo con el examen final.

\section{Evaluación y conclusiones}

Esta experiencia se realizó de form a similar en el curso anterior. $\mathrm{S}$ in embargo, se ha implementado la cocreación de las rúbricas c on los es tudiantes, la división de este instrumento en fases, el trabajo d e sus dife rentes partes a lo largo de la asignatura enfocado al trab ajo, la petición d e entregas parciales con la im plementación de actividades metacognitivas y autorregulatorias, la provisión de retroalim entación en las clases a través de trab ajo por es taciones y la solicitud de un listado de tareas y responsables con un modelo de tareas pendientes-en proceso-terminadas a través de la App Trello. Se podrá hacer un a evaluación en profundidad de la ex periencia en el congreso una vez concluida la asignatura. 
Se obtendrán datos para evaluar la expe riencia mediante la reunión con cada grupo para la revisión de la entrega parcia 1, la cum plimentación de un cuestionario sobre el empleo de las rúbricas y la opini ón sobre el resto de los recursos em pleados (entrega parcial, empleo de la app Trello, etc.).

\section{Referencias}

Fraile, J., Panadero, E., \& Pardo, R. (2017). Co-creating rubrics: The effects on sel fregulated learning, self-efficacy an d performance of establishing assessm ent criteria with students. Studies in Educational Evaluation, 53, 69-76.

Kostons, D., van Gog, T., \& Paas, F. ( 2012). Training self-a ssessment and taskselection skills: A cognitiv e approach to improving self-regulated learn ing. Learning and Instruction, 22(2), 121-132.

Panadero, E., \& Jonsson, A. (2013). The use of scoring rubrics for formative assessment purposes revisited: A review. Educational Research Review, 9, 129-144. http://doi.org/10.1016/j.edurev.2013.01.002 